\title{
Sketchy Tweets: Ten Minute Conjectures in Graph Theory
}

\section{Anthony Bonato and Richard J. Nowakowski}

G omments by Richard Hamming in his address You and Your Research [16] resonated with us. On the one hand, Hamming says,

"What are the most important problems in your field?"

which suggests working on hard problems. Yet on the other, he exhorts us to

"Plant the little acorns from which mighty oak trees grow."

Following this advice, we should look over the big questions, then doodle and sketch out some approaches. If you are an expert, then this is easy to do, but most people do not want to wait to become an expert before looking at interesting problems. Graph theory, our area of expertise, has many hard-to-solve questions. Some hark back to the recreational roots of the area yet still keep their mystery. These "acorns" can be planted on the backs of envelopes, on a blackboard, and over a coffee.

Our goal is to collect some of these conjectures-arguably some of the most intriguing-in one place. We present ten conjectures in graph theory, and you can read about each one in at most ten minutes. As we live in the era of Twitter, all the conjectures we state are 140 characters or fewer (so "minute" here has a double meaning). We might even call these sketchy tweets, as we present examples for each conjecture that you can doodle on as you read.

Hamming also references ambiguity: good researchers can work both on proving and disproving the same statement, so we approach the conjectures with an open mind. He also mentions that a good approach is to reframe the problem, and change the point of view. One example from Vizing's
Conjecture (which is discussed as our second-to-last conjecture in the following text), is the three-page paper [2] which, with a new way of thinking, reduced most of the published work of twenty years to a corollary of its main result!

Given the size of modern graph theory, with its many smaller subfields (such as structural graph theory, random graphs, topological graph theory, graph algorithms, spectral graph theory, graph minors, and graph homomorphisms, to name a few), it would be impossible to list all, or even the bulk of the conjectures in the field. We are content instead to focus on a few family jewels, which have an intrinsic beauty and have provided some challenges for graph-theorists for at least two decades. There is something for everyone here, from undergraduate students taking their first course in graph theory, to seasoned researchers in the field. For additional reading on problems and conjectures in graph theory and other fields, see the Open Problem Garden maintained by IRMACS at Simon Fraser University [24].

We consider only finite and undirected graphs, with no multiple edges or loops (unless otherwise stated). We assume the reader has some basic familiarity with graphs and their terminology, including notions such as cycles, paths, complete graphs, complete bipartite graphs, vertex degrees, and connected graphs. We use the notation $C_{n}$ for the cycle with $n$ vertices, $P_{n}$ for the path with $n$ vertices, and $K_{n}$ for the complete graph with $n$ vertices. The complete bipartite graph with $m$ and $n$ vertices of the respective colors is denoted by $K_{m, n}$.

All the background we need can be found in any text in graph theory, such as those of Diestel [9] and West [41], or online (see for example [42]). 
For a graph $G$, we write $V(G)$ for its vertex set, and $E(G)$ for its edge set. If two vertices are joined by an edge, then we say they are adjacent. The order of a graph is the cardinality of its vertex set.

\section{The Conjectures}

Some conjectures we present (such as Meyniel's) are less known and deserve more exposure, whereas others (such as Hadwiger's) are better known. We provide no justification for our bias toward one problem over another, so we apologize upfront if your favorite conjecture is missing.

We present each conjecture using minimal technical jargon. To shorten the number of references to partial results, we cite surveys wherever possible. We always cite the original authors of the problem. The conjectures we present have spawned enormous amounts of work on related problems and concepts, which in the present article can only be hinted at. For example, a quick check of Google Scholar or MathSciNet will reveal many thousands of papers related to the topic of one of the ten conjectures we present. Some of these works are discussed in the surveys.

All the conjectures here are considered difficult, having remained unsolved for many years. We do not rank the conjectures in order of difficulty. To show no preference among the problems, we present the conjectures in alphabetical order.

\section{Double the Fun}

There is an old puzzle, found in many books that feature "pencil-and-paper" problems, of attempting to trace a diagram without lifting the pen off the paper or retracing any part of the figure. Euler in his famous 1736 solution to the Königsberg bridge problem, essentially found when this can be done. The problem can be restated as covering the diagram (represented by a graph) with a cycle. Such graphs are now called Eulerian; the connected Eulerian graphs are those in which every vertex has even degree. (We note that Euler did not prove the characterization of Eulerian graphs; the first proof was published by Hierholzer [18].) Our first conjecture may be thought of as a generalization of this kind of problem to graphs with some vertices of odd degree.

A bridge is an edge whose deletion disconnects the graph. A graph with no bridges is bridgeless. For example, each edge of a tree is a bridge.

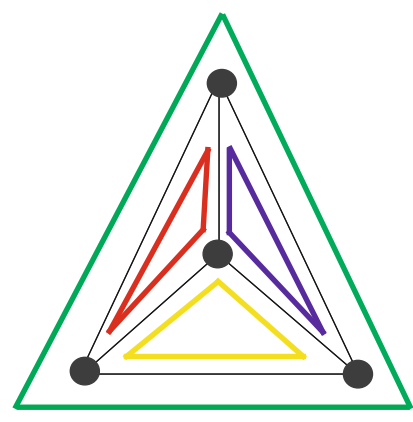

Figure I. A CDC in $K_{4}$, with the cycles in different colors. What would be a CDC of $K_{5}$ ?

Cycle Double Cover Conjecture: For every bridgeless graph, there is a list of cycles so that every edge appears in exactly two cycles.

A list of cycles as in the conjecture is called a CDC. See Figure 1 for an example. Note that the list may have repeated cycles, as is the case with $C_{n}$. The conjecture was formulated independently by Szekeres in 1973 [34] and Seymour in 1979 [30]. See the survey [20] and book [45] for additional background and references on the conjecture.

The conjecture has connections to embeddings of graphs on surfaces; that is, drawings of graphs on different surfaces so that no two edges cross. The simplest case is the family of planar graphs, which have an embedding in the plane (see Hadwiger's Conjecture for more on planar graphs). If each face in the embedding corresponds to a cycle in the graph, then the faces form a CDC as in Figure 1, as is true for all connected, bridgeless, planar graphs. That there is an embedding in some surface where each face corresponds to a cycle is the Strong Embedding Conjecture, which is a stronger conjecture than the Cycle Double Cover Conjecture.

In the other direction, much is known about the smallest counterexample, if it exists: every vertex has degree 3 , it is not 3-edge-colorable (i.e., there is no coloring of the edges with three colors so that no edge is incident with an edge of the same color), it is cyclically 4-connected (i.e., every partition of the vertices into two parts with a cycle in each part has at least four edges that join the parts), and the smallest cycle has length at least 10 . A connected, bridgeless, cubic graph that is not 3-edge-colorable is called a

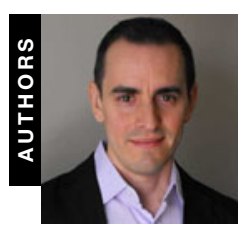

ANTHONY BONATO is Chair of the Department of Mathematics at Ryerson. His research is mostly on graph theory, including its applications to real-world complex networks. He is an Editor-in-Chief of the journal Internet Mathematics.

Department of Mathematics
Ryerson University
Toronto, M5B 2K3
Canada
e-mail: abonato@ryerson.ca

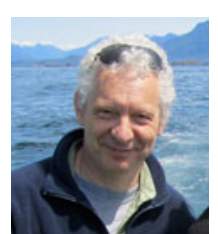

RICHARD J. NOWAKOWSKI is a Professor at Dalhousie and is one of the leaders of the Graphs and Games Group there. He studies especially combinatorial game theory-learning, at least, how to lose well-and graph theory.

Department of Mathematics and Statistics

Dalhousie University

Halifax, (NS) B3J 3H5

Canada

e-mail: rjn@mathstat.dal.ca 


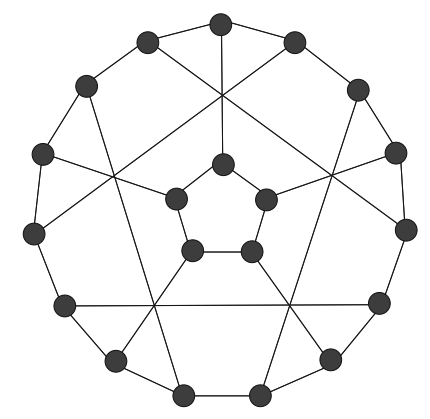

Figure 2. The flower snark $J_{5}$.

snark. Another conjecture says that there are not any snarks with the smallest cycle length at least 10. See Figure 2 for an example of a snark.

A stronger conjecture than the Cycle Double Cover Conjecture is the Small Cycle Double Cover Conjecture: every bridgeless graph on $n$ vertices has a CDC of size at most $n-1$.

\section{Party, But Know Your Limits}

Frank Ramsey, who died at the early age of 26, wrote a paper [26] in mathematical logic that has gone on to have applications in many fields, including graph theory. To motivate Ramsey numbers, consider a party with six people, some pairs of whom are friends, and some of whom are strangers. It is not hard to show that among six people we can always find three mutual friends or three mutual strangers. Readers should convince themselves that in smaller parties this property is not always satisfied.

Ramsey numbers generalize this setting from three to $n$ mutual friends or strangers. For a positive integer $n$, define the $n$th Ramsey number, written $R(n)$, to be the minimum integer $r$ such that any coloring of the edges of $K_{r}$ with red or blue (red joins friends and blue joins strangers) results in a complete subgraph of order $n$ whose edges all have the same color.

It is not immediately clear whether the Ramsey numbers even exist. Calculating them directly is hard; whereas $R(4)=18$, the value of $R(5)$ is unknown (although it is between 43 and 49; see [25] for a dynamic survey of the known small Ramsey numbers). We must be content with lower and upper bounds. An inductive argument gives $R(n) \leq\left(\begin{array}{c}2 n-2 \\ n-1\end{array}\right)$. In an early application of the probabilistic method, Erdős [11] proved the lower bound

$$
(1+o(1)) \frac{1}{e \sqrt{2}} n 2^{n / 2} \leq R(n),
$$

which has not been substantially improved to this day (Spencer [32] improved the constant $\frac{1}{\sqrt{2}}$ to $\sqrt{2}$ ). The best known upper bound for $R(n)$, which is far apart from the known lower bounds, was given by Thomason [36]:

$$
R(n) \leq n^{-1 / 2+c / \sqrt{\log n}}\left(\begin{array}{c}
2 n-2 \\
n-1
\end{array}\right)
$$

Erdős in 1947 posed the following asymptotic conjecture, and it remains one of the major topics in Ramsey numbers.

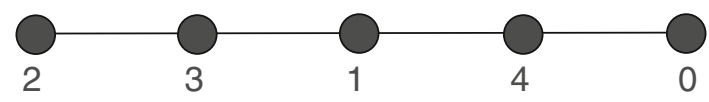

Figure 3. The path $P_{5}$ is graceful. Show that all the paths $P_{n}$ are graceful.

Erdős' Ramsey Number Conjecture: $\lim _{n \rightarrow \infty} R(n)^{1 / n}$ exists.

Solve this conjecture and you will be awarded $\$ 100$. However, this is a ridiculously difficult way to make $\$ 100$ ! From the bounds stated previously, if the limit exists, then it is between $\sqrt{2}$ and 4 (finding the value of the limit is worth $\$ 250$ ). Hence, we can think of the conjecture as a way to understand better which bound for $R(n)$ stated previously is more accurate. For more on Erdös and his questions about Ramsey numbers, see [8].

\section{Saving Grace}

We next consider a graph-labelling problem, where vertices or edges are labelled by numbers subject to some constraints. A graph is graceful if the vertices can be assigned numbers from among $0,1, \ldots, m$, where $m$ is the number of edges, so that the differences along the edges are precisely $1,2, \ldots, m$. Graceful graphs have received ample attention in the literature. See Figure 3 for an example.

Graceful labellings were introduced by Rosa under the name of $\beta$-labellings and were renamed "graceful" by Golomb.

The following conjecture is now sometimes called the Ringel-Kotzig Conjecture (because, if the conjecture were true, it would imply conjectures of both authors on certain decompositions of complete graphs).

Graceful Tree Conjecture: Every tree is graceful.

More than 200 papers have been written on proving special cases of this conjecture, and a bewildering number of variants on graceful labellings have been proposed and studied. See the dynamic survey of Gallian [13] for further background and references on graceful (and other) labellings. Kotzig labelled the collective work on proving the conjecture a "disease." A few of the classes of trees where we know the conjecture holds are: caterpillars (a caterpillar is a tree whose nonleaf vertices form a path), trees with at most four leaves, trees with diameter at most 5 , and trees with at most 35 vertices.

Much of the research on the conjecture tries to settle it in the affirmative. One class of trees where the conjecture remains open are lobsters (those where the removal of the leaves gives a caterpillar).

\section{No Minors Allowed}

Coloring has both fascinated and perplexed graph-theorists since the early days of the field. The chromatic number of $G$, written $\chi(G)$, is the minimum number of colors in a vertex labelling such that adjacent vertices receive distinct colors; that is, the minimum $k$ such that $G$ is $k$-colorable. The most famous theorem proved so far in graph theory is the Four-Color theorem [1], which states that every planar graph is 4-colorable. All known proofs of this fact are computer-assisted. 
A graph is a minor of $G$ if it results by repeatedly performing one of the following operations: i) deleting a vertex, ii) deleting an edge, or iii) contracting an edge (i.e., shrinking an edge to a vertex and preserving adjacencies and nonadjacencies with vertices outside the edge). A beautiful result of Wagner [40] states that a graph is planar if and only if it does not have $K_{5}$ or $K_{3,3}$ as a minor. The reader can show that the Petersen graph (see Figure 8 ) has $K_{5}$ as a minor, and hence, is not planar.

Hadwiger's Conjecture, dating back to 1943 [15], relates graph coloring to minors.

Hadwiger's Conjecture: For $m \geq 2$, a graph not having $K_{m}$ as a minor is $(m-1)$-colorable.

Hadwiger's Conjecture is open for $m \geq 7$. The startling case for small $m$ is $m=5$, which was shown by Wagner [40] to reduce to the Four-Color theorem. Hence, Hadwiger's Conjecture may be viewed as a broad generalization of that theorem. The case $m=6$ was settled by Robertson, Seymour, and Thomas [28] by showing that a minimal counterexample to the conjecture is planar after the removal of one vertex (so this also reduces to the Four-Color Theorem).

The cases $m=2$ and 3 are elementary (for example, a graph without $K_{2}$ as a minor has no edges, and a graph bit having $K_{3}$ as a minor is a forest). Dirac [10] and Hadwiger [15] proved the case $m=4$, by showing that graphs not having $K_{4}$ as a minor have a vertex of degree at most 2 and, hence, can be 3-colored using a greedy algorithm. Although the case $m=7$ is open, in 2005 Kawarabayashi and Toft [21] proved that any 7 -chromatic graph has $K_{7}$ or $K_{4,4}$ as a minor.

\section{$X$ Marks the Spot}

As with Hadwiger's Conjecture, our next conjecture also deals with coloring but adds graph products to the mix. All the references in this section can be found in three surveys on the conjecture: [29, 35, 46].

A graph product makes new graphs from old. We consider one of the best known products: the categorical product (which is also referred to as the tensor or Kronecker product). For graphs $G$ and $H$, define $G \times H$ to have vertex set $V(G) \times V(H)$, with $(a, b)$ adjacent to $(c, d)$ if $a$ is joined to $c$ in $G$, and $b$ is joined to $d$ in $H$. See Figure 4 , which motivates the notation for this product.

Hedetniemi's Conjecture yields a simple formula for the chromatic number of the categorical product; it was posed by him in 1966 [17] while he was a graduate student.

Hedetniemi's Conjecture: For graphs $G$ and $H$,

$$
\chi(G \times H)=\min \{\chi(G), \chi(H)\} .
$$

The conjecture was stated independently by Burr, Erdős, and Lovász in 1976. Most experts think the conjecture is

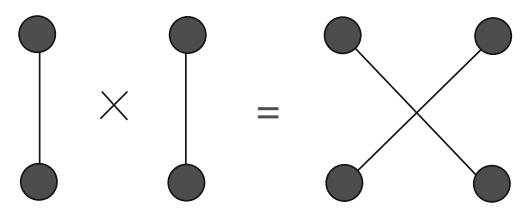

Figure 4. The graph $K_{2} \times K_{2}$.

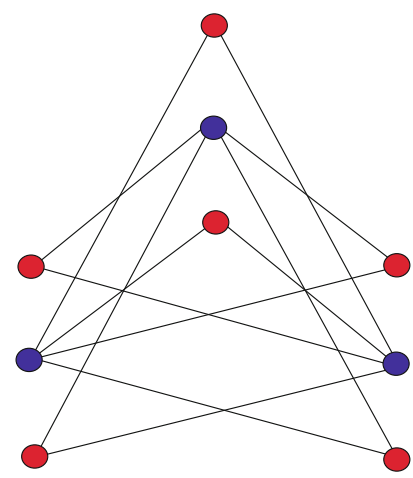

Figure 5. The graph $P_{3} \times K_{3}$ is 2-colorable.

true. For starters, $G \times H$ may be visualized as replacing each vertex $v$ of $G$ by a copy of the vertices of $H$. Label these vertices as $(v, b)$. Then add the edges $(v, b)(w, j)$ just if $v$ is adjacent to $w$ and $b$ is adjacent to $j$. See Figure 5. Now take a proper coloring of $G$. For each vertex $v$ of $G$, color all vertices $(v, b)$ with the same color as $v$. Since $(v, b)$ and $\left(v, b^{\prime}\right)$ are not adjacent, this is also a proper coloring of $G \times H$. Hence, $\chi(G \times H) \leq \chi(G)$. The same construction, but considering vertices of $H$, gives $\chi(G \times H) \leq \chi(H)$.

The conjecture has a convenient restatement that is often used. For a positive integer $n$, let $H(n)$ denote the following statement:

$$
\text { If } \chi(G \times H)=n \text {, then either } \chi(G)=n \text { or } \chi(H)=n \text {. }
$$

Hedetniemi's Conjecture is equivalent to $H(n)$ being true for all $n \geq 1$, which permits an incremental approach. Indeed, it is not too difficult to show that $H(1)$ and $H(2)$ are true. El-Zahar and Sauer proved in 1985 that $H(3)$ is true, but little is known about $H(n)$ for $n>3$.

Burr, Erdős, and Lovász in their 1976 paper showed that if $G$ is a graph in which every vertex lies in a complete subgraph of order $n$, and $H$ is a connected graph with $\chi(G \times H)=n$, then $\min \{\chi(G), \chi(H)\}=n$. (In Figure 5, we have that $n=2)$. This is not too surprising, since the presence of $K_{n}$ in $G$ is a trivial reason why $\chi(G) \geq n$. Proofs of the conjecture, or the search for a counterexample, therefore must consider graphs that have large chromatic number and small complete subgraphs.

The strangest result arising out of the work on the conjecture has to do with a special case. Define the function

$$
g(n)=\min \{\chi(G \times H): \chi(G)=\chi(H)=n\} .
$$

It is known that $g(1)=1, g(2)=2, g(3)=3$, and $g(4)=4$. Several authors discovered the striking fact that either $g$ is unbounded or $g(n) \leq 9$ for all $n$. Since Hedetniemi's Conjecture has received a lot of attention over the past 45 years, if there were a counterexample surely it would have been found by now!

We mention in passing (and without explanation of the jargon!) that Hedetniemi's Conjecture is equivalent to the meet-irreducibility of the complete graphs in the lattice of cores. For this reason, the conjecture is of ample interest not only to experts in graph coloring, but also to those working on graph homomorphisms. 


\section{The Long Arm of the Law}

Many of us played games such as Cops and Robbers (or other pursuit games) as children, and our next conjecture considers such a game played on graphs. In the graph game of Cops and Robbers there are two players, a team of cops and a robber, who move from vertex to vertex along edges in the graph or can pass. The game is played with alternate moves of the players. The cops move first, by choosing some set of vertices for their team to occupy. The robber then chooses a vertex. The cops win if eventually they capture or land on the vertex with the robber; the robber wins if he can indefinitely evade capture. The game has perfect information, in the sense that both players can see and remember each other's moves. Placing a cop on each vertex provides an easy win for the cops. The minimum number of cops needed to win the game is the cop number of a graph. The reader may verify that the cop number of the snark $J_{5}$ in Figure 2 is 3 .

As the cop number of a disconnected graph is the sum of the cop numbers of its components, it is sensible to consider only connected graphs. For functions $f$ and $g$ on positive integers taking positive real values, we write $f=O(g)$ to mean that there is a constant $d$, such that for large enough $n, f(n) \leq d g(n)$.

Meyniel's Conjecture: If $G$ is a connected graph, then

$$
c(G)=O(\sqrt{|V(G)|} .
$$

Meyniel's Conjecture states that about a constant multiple of $\sqrt{n}$ many cops are sufficient to capture the robber in a connected graph of order $n$ (and there are examples of graphs needing this many cops). Aigner and Fromme in 1984 proved that the cop number of a planar graph is at most 3.

Meyniel's Conjecture may be one of the lesser known unsolved conjectures in graph theory, but it has received a fair bit of recent attention. For further background on the conjecture, see Chapter 3 of the book [3]. Meyniel's Conjecture was communicated by Frankl [12], who could only prove that

$$
c(G)=O\left(n \frac{\log \log n}{\log n}\right) .
$$

To date the best available general bound is the following, recently discovered by three independent sets of researchers:

$$
c(G)=O\left(\frac{n}{2^{(1-o(1)) \sqrt{\log _{2} n}}}\right) .
$$

Even to prove that $c(G)=O\left(n^{1-\varepsilon}\right)$ for some positive $\varepsilon$ is open! The conjecture was settled for bipartite graphs with diameter 3, and Andreae proved it is true in graph classes formed by avoiding a fixed graph as a minor (in fact, the cop number is bounded by a constant in such graphs); see Chapter 3 of [3].

\section{House of Cards}

The Reconstruction Conjecture has proved to be notoriously difficult and suggests how much more there is to learn about graphs. The deck of a graph $G$ is the multiset

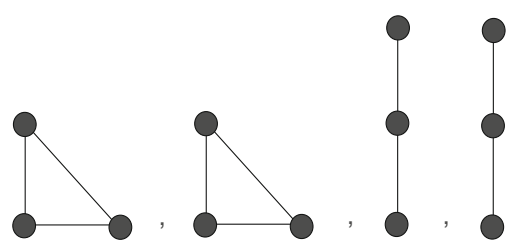

Figure 6. Which graph has this deck?

consisting of all subgraphs of $G$ formed by deleting a vertex. Each such vertex-deleted subgraph is a card. See Figure 6 for an example.

The conjecture was posed independently by Kelly in 1957 [22] and Ulam in 1960 [38].

Reconstruction Conjecture: If two graphs with at least three vertices have the same deck, then they are isomorphic.

It is easy to see that $K_{2}$ and its complement have the same deck: hence the modest requirement on the order of the graph. Given a deck, we immediately know the order of $G$, and some thought yields the number of edges and the degrees of all the vertices. For references to results on the conjecture, see the survey [4]. Kelly proved that disconnected graphs, trees, and regular graphs are reconstructible from their deck. McKay showed that the conjecture is true for all graphs with at most 11 vertices. The conjecture also holds for outerplanar graphs. Bollobás proved that with probability tending to 1 as $n$ tends to infinity, there exist three cards that determine the graph. Surprisingly, the conjecture remains open for planar graphs.

\section{Go with the Flow}

We may view the edges of a graph as a series of pipes transporting some liquid (or electric current, or information) between nodes. Usually edges have a maximum capacity for carrying materials, and what enters a node must equal what must come out. Further, these flows, as they are called, usually move in one direction, so some orientation must be assigned to the edges of the network. Flows have deep connections to the Four-Color Theorem, and Tutte's conjecture on flows extends these connections beyond the context of planar graphs.

To be more precise, an integer flow on a graph is a pair consisting of an orientation of the graph and an assignment of integer weights to the edges such that for each vertex, the total weight on exiting edges equals the total weight on entering edges. It is a $k$-flow if all weights have absolute value less than $k$, and it is nowhere-zero if weight 0 is never used. See Figure 7 . Note that every $k$-flow is a $(k+1)$-flow.

Nowhere-zero $k$-flows were introduced by Tutte [37] as a generalization of face-coloring problems in planar graphs (where we color the faces so that no adjacent faces receive
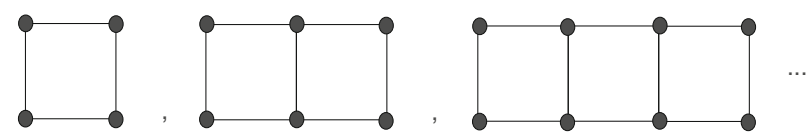

Figure 7. Find nowhere-zero 4-flows for the graphs in this sequence. Do CDCs help? 


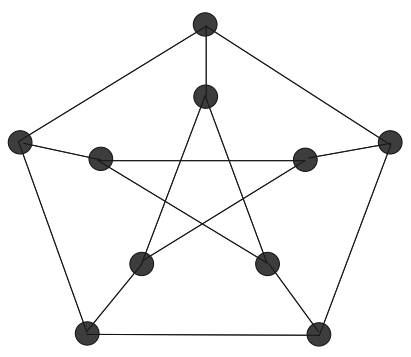

Figure 8. The Petersen graph.

the same color). The famous Four-Color Theorem is equivalent to saying that every planar bridgeless graph has a nowhere-zero 4-flow. Unfortunately, this result cannot be extended to arbitrary bridgeless graphs, since the Petersen graph has no nowhere-zero 4-flow. See Figure 8.

Tutte in 1954 therefore considered 5-flows instead and conjectured the following in [37].

Tutte's 5-Flow Conjecture: Every bridgeless graph has a nowhere-zero 5-flow.

The conjecture holds for planar graphs, using the duality between flows and coloring and the Five-Color Theorem (every planar graph is 5-colorable). We may therefore view the 5-flow conjecture as a generalization of the Five-Color Theorem to graphs that are not planar.

Jaeger [19] proved that every bridgeless graph has a nowhere-zero 8-flow. Seymour [31] improved upon this result by showing that bridgeless graphs have nowhere-zero 6-flows. Celmins [6] proved that a smallest counterexample to the conjecture must be a cyclically 5-edge-connected snark with girth at least 7 (see also the Cycle Double Cover Conjecture). The conjecture can be reduced to the 3-regular case, and Steinberg [33] proved the conjecture for graphs that embed on the projective plane.

\section{You Are So Square}

We state another conjecture about products, this time related to domination. (No, not the other kind!) For graphs $G$ and $H$, define the Cartesian product of $G$ and $H$, written $G \square H$, to have vertex set $V(G) \times V(H)$, with $(a, b)$ adjacent to $(c, d)$ if $a=c$ and $b$ is joined to $d$ in $H$, or if $b=d$ and $a$ is joined to $c$ in $G$. See Figure 9, which motivates the notation for this product.

In a graph $G$, a set $S$ of vertices is a dominating set if every vertex not in $S$ has a neighbour in $S$. The domination number of $G$, written $\gamma(G)$, is the minimum size of a dominating set. For example, see Figure 10, where we note that $\gamma\left(C_{4} \square C_{4}\right)=\gamma\left(C_{4}\right) \gamma\left(C_{4}\right)=4$.

The following was proposed by Vizing in 1968 [39].

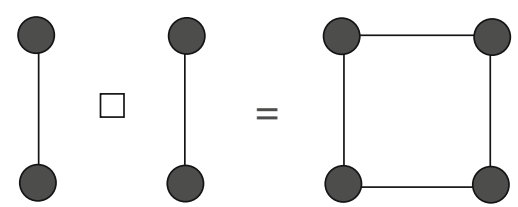

Figure 9. The graph $K_{2} \square K_{2}$.

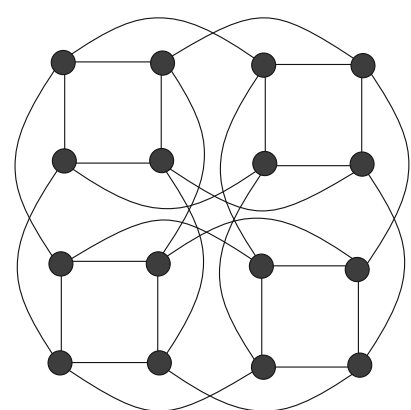

Figure 10. Find a dominating set of size 4 in $C_{4} \square C_{4}$.

Vizing's Conjecture: For graphs $G$ and $H$,

$$
\gamma(G \square H) \geq \gamma(G) \gamma(H) .
$$

This is a conjecture everyone thinks is true. All the references that follow appear in [5].

An important theorem from 1979 was not appreciated (or was completely overlooked) for the next 16 years. During some collaboration of Rall and the second author of this article, they noticed Math Review MR0544028 of a paper by Barcalkin and German [2]. From that brief description, in a couple of days Hartnell and Rall were able to reconstruct the mathematical arguments of the paper (rather than translate the paper, which was in Russian). The results in [2] reduced much of the work from 1968 through 1996 to a corollary of their main theorem!

As with several of the conjectures considered so far, many experts think that if the conjecture were false then a minimal counterexample would have been found. But since a proof has not been found, where would one look for a counterexample? For example, a minimal counterexample to Vizing's conjecture must have domination number larger than 3; adding an edge between two nonadjacent vertices decreases the domination number; and every vertex belongs to a minimum dominating set.

\section{Don't Get Cross}

Our final conjecture has its origins in a 1940s labor camp in Budapest. The famous mathematician Turán was imprisoned there, watching trucks move bricks along rails from kilns to storage areas. Every once in a while, two trucks would cross each other's paths and the bricks would come crashing down. No doubt as a kind of liberation from the monotony, Turán began thinking about minimizing the crossings of the trucks, assuming the general situation that there were $m$ kilns and $n$ trucks. For more on the history of the problem see [47].

We may formalize Turán's problem in the following way. The crossing number of $G$, written $\operatorname{cr}(G)$, is the minimum number of pairwise crossings of edges in a drawing of $G$ in the plane. Some readers may recall the Three Utilities Problem, which reduces to showing that $K_{3,3}$ has crossing number 1 .

Crossing numbers tend to be hard to calculate exactly, because of the exponentially many drawings any given graph may possess. The following conjecture is named after Zarankiewicz who published a flawed proof of it [44], but it is also called Turán's Brick Factory Conjecture (see [14] for a survey of the history of the conjecture). 


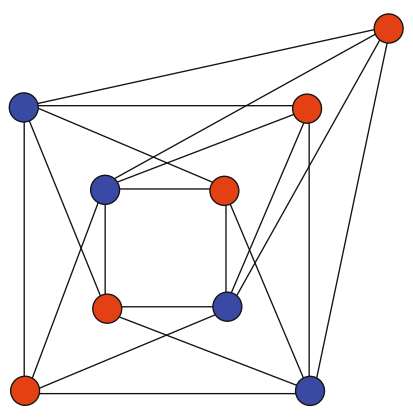

Figure II. A drawing of $K_{4,5}$ with 8 crossings, which is the minimum number possible.

\section{Zarankiewicz's Conjecture:}

$$
\operatorname{cr}\left(K_{m, n}\right)=\left\lfloor\frac{m}{2}\right\rfloor\left\lfloor\frac{m-1}{2}\right\rfloor\left\lfloor\frac{n}{2}\right\rfloor\left\lfloor\frac{n-1}{2}\right\rfloor .
$$

The best exact result on the conjecture was published by Kleitman in 1970 [23] who confirmed it for $n \leq 6$. Kleitman also proved that the smallest counterexample, if it exists, must occur for $m$ and $n$ odd. Woodall [43] computed the crossing numbers $\operatorname{cr}\left(K_{7,7}\right)=81$ and $\operatorname{cr}\left(K_{7,9}\right)=144$. Hence, the smallest unsolved cases are for $K_{7,11}$ and $K_{9,9}$. As $\left\lfloor\frac{4}{2}\right\rfloor\left\lfloor\frac{3}{2}\right\rfloor\left\lfloor\frac{5}{2}\right\rfloor\left\lfloor\frac{4}{2}\right\rfloor=8$, the drawing in Figure 11 achieves the conjectured bound.

A recent result in [7] states that if for a fixed $m$, the conjecture holds for all values $n$ smaller than some constant depending on $m$, then the conjecture holds for all $n$. Hence, for each $m$ there is an algorithm that verifies the conjecture for all $n$ or gives a counterexample.

\section{Epilogue}

Now it is your turn to finish this survey and solve (or partially solve) one or more of these conjectures in graph theory. And when you are done with those, we have a few others that might keep you busy, such as Barnette's Conjecture, the Berge-Fulkerson Conjecture, the Erdös-Sós Conjecture, the Middle Levels Conjecture, or Sheehan's Conjecture.

Our version of another Hamming quote [16] provides our parting words:

"Go forth, then, and doodle great work!"

\section{ACKNOWLEDGMENTS}

We would like to thank the anonymous referees for several suggestions that improved the quality and readability of the paper.

\section{REFERENCES}

[1] K. Appel, W. Haken, J. Koch, Every planar map is four colorable, Illinois J. Math. 21 (1977), 439-567.

[2] A. M. Barcalkin, L. F. German, The external stability number of the Cartesian product of graphs, Bul. Akad. Stiinte RSS Moldoven, 94 (1979), 5-8.

[3] A. Bonato, R. J. Nowakowski, The Game of Cops and Robbers on Graphs, American Mathematical Society, Providence, Rhode Island, 2011.
[4] J. A. Bondy, A Graph Reconstructor's Manual, Surveys in Combinatorics, 1991 (Guildford, 1991), London Mathematical Society Lecture Note Series, 166, Cambridge University Press, Cambridge, 1991, pp. 221-252.

[5] B. Brešar, P. Dorbec, W. Goddard, B. L. Hartnell, M. A. Henning, S. Klavžar, D. F. Rall, Vizing's conjecture: a survey and recent results, J. Graph Theory 1(2012), 46-76.

[6] U. Celmins, On cubic graphs that do not have an edge-3coloring, Ph.D. Thesis, Department of Combinatorics and Optimization, University of Waterloo, Waterloo, Canada, 1984.

[7] R. Christian, B. R. Richter, G. Salazar, Zarankiewicz's conjecture is finite for each fixed $m$, Preprint 2011.

[8] F. R. K. Chung, Open problems of Paul Erdős in graph theory, J. Graph Theory 25 (1997), 3-36.

[9] R. Diestel, Graph Theory, Springer-Verlag, New York, 2000.

[10] G. A. Dirac, A property of 4-chromatic graphs and some remarks on critical graphs, J. Lond. Math. Soc. 27 (1952), 85-92.

[11] P. Erdős, Some remarks on the theory of graphs, Bull. Amer. Math. Soc. 53 (1947), 292-294.

[12] P. Frankl, Cops and robbers in graphs with large girth and Cayley graphs, Discrete Appl. Math. 17 (1987), 301-305.

[13] J. A. Gallian, A dynamic survey of graph labeling, Electr. J. Comb. DS6 (2002).

[14] R. K. Guy, The Decline and Fall of Zarankiewicz's Theorem, Proof Techniques in Graph Theory (Proc. Second Ann Arbor Graph Theory Conf., Ann Arbor, Mich., 1968), Academic Press, New York, 1969, pp. 63-69.

[15] H. Hadwiger, Über eine Klassifikation der Streckenkomplexe, Vierteljschr. Naturforsch. Ges. Zürich 88 (1943), 133-143.

[16] R. Hamming, You and Your Research. Accessed December 14, 2011. http://www.cs.virginia.edu/ robins/YouAndYourResearch.html.

[17] S. Hedetniemi, Homomorphisms of graphs and automata, Technical Report 03105-44-T, 1966.

[18] C. Hierholzer, Ueber die Möglichkeit, einen Linienzug ohne Wiederholung und ohne Unterbrechung zu umfahren, Mathematische Annalen 6 (1873), 30-32.

[19] F. Jaeger, Flows and generalized coloring theorems in graphs, J. Combin. Theory Ser. B 26 (1979), 205-216.

[20] F. Jaeger, A survey of the cycle double cover conjecture, Ann. Discrete Math. 27 (1985), 1-12.

[21] K. Kawarabayashi, B. Toft, Any 7-chromatic graph has $K_{7}$ or $K_{4,4}$ as a minor, Combinatorica 25 (2005), 327-353.

[22] P. J. Kelly, A congruence theorem for trees, Pacific J. Math. 7 (1957), 961-968.

[23] D. J. Kleitman, The crossing number of $K_{5, n}, J$. Combinatorial Theory 9 (1970), 315-323.

[24] Open Problem Garden. Accessed August 23, 2011. http://garden.irmacs.sfu.ca/.

[25] S. P. Radziszowski, Small Ramsey numbers, Electr. J. Comb., Dynamic Survey DS1.

[26] F. P. Ramsey, On a problem of formal logic, Proc. Lond. Math. Soc. 30 (1930), 264-286.

[27] G. Ringel, problem 25, In: Theory of Graphs and Its Applications, Proc. Symp. Smolenice, 1963, G/A Czech. Acad. Sci. 162 (1964).

[28] N. Robertson, P. Seymour, R. Thomas, Hadwiger's conjecture for $K_{6}$-free graphs, Combinatorica 13 (1993), 279-361. 
[29] N. Sauer, Hedetniemi's conjecture: a survey, Discrete Math. 229 (2001), 261-292.

[30] P. D. Seymour, Sums of circuits, In: Graph Theory and Related Topics, J. A. Bondy and U. S. R. Murty (eds.), Academic Press, New York (1979), pp. 341-355.

[31] P. D. Seymour, Nowhere-zero 6-flows, J. Combin. Theory Ser. B 30 (1981), 130-135.

[32] J. H. Spencer, Ramsey's theorem-a new lower bound, J. Combin. Theory Ser. A 18 (1975), 108-115.

[33] R. Steinberg, Tutte's 5-flow conjecture for the projective plane, J. Graph Theory 8 (1984), 277-289.

[34] G. Szekeres, Polyhedral decomposition of cubic graphs, Bull. Aust. Math. Soc. 8 (1973), 367-387.

[35] C. Tardif, Hedetniemi's conjecture, 40 years later, Graph Theory Notes N.Y., 54 (2008), 46-57.

[36] A. Thomason, An upper bound for some Ramsey numbers, J. Graph Theory 12 (1988), 509-517.

[37] W. T. Tutte, A contribution to the theory of chromatic polynomials, Canad. J. Math. 6 (1954), 80-91.

[38] S. M. Ulam, A Collection of Mathematical Problems, Wiley, New York, 1960.
[39] V. G. Vizing, Some unsolved problems in graph theory, Uspehi Mat. Nauk 23 (1968), 117-134.

[40] K. Wagner, Über eine Eigenschaft der ebenen Komplexe, Math. Ann. 114 (1937), 570-590.

[41] D. B. West, Introduction to Graph Theory, 2nd edition, Prentice Hall, 2001.

[42] Wikipedia: Glossary of Graph Theory, Accessed December 14, 2011. http://en.wikipedia.org/wiki/Glossary of graph theory.

[43] D. R. Woodall, Cyclic order graphs and Zarankiewicz's crossing number conjecture, J. Graph Theory 17 (1993), 657-671.

[44] K. Zarankiewicz, On a problem of P. Turán concerning graphs, Fund. Math. 41 (1954), 137-145.

[45] C. Q. Zhang, Integer Flows and Cycle Covers of Graphs, Marcel Dekker Inc., New York, 1997.

[46] X. Zhu, A survey on Hedetniemi's conjecture, Taiwanese J. Math. 2 (1998), 1-24.

[47] L. Beineke, R. Wilson, The early history of the brick factory problem, Math. Int. 32 (2010), no. 2, 41-48. 